\title{
THALIDOMIDE AND CONGENITAL ABNORMALITIES
}

BY

S. P. WARD, M.B., B.S.

Department of Clinical Pathology, Staffordshire General Infirmary, Stafford

The continued interest by medical observers and the preoccupation of the daily press with the effects of thalidomide on the foetus encourages yet another account of congenital abnormalities believed to be due to thalidomide ("distaval") taken during pregnancy.

Although the account which follows dates from February, 1962. publication was delayed because all the medical literature at that time suggested a direct relation between the time in pregnancy at which thalidomide was taken and the type of malformation produced. implying that arrest of development occurs in organs actively forming in the weeks when the drug is being taken.

In this case the mother had taken thalidomide for about two months before but for only three weeks after conception. This made correlation with the limb defects noted in her infant difficult, as the limb buds do not start to form until the sixth to the seventh week of intrauterine life. It was thought that the suspicion of these abnormalities being due to thalidomide was not strong enough to warrant publication.

However, a recent article by Woollam (1962) puts forward a suggestion which once again implicates thalidomide as a cause for the foetal abnormalities produced in the case reported below. He states: "The time at which an agent is administered does not necessarily coincide with the time at which it exerts its deleterious effects. It may indeed be necessary for administration to begin before pregnancy in order to achieve a sufficient accumulation of teratogen by the critical period.'

\section{Case Report}

A woman of 35 , in her first pregnancy after 12 years of marriage, took thalidomide as a sedative for two months prior to conception and for the first three weeks only of

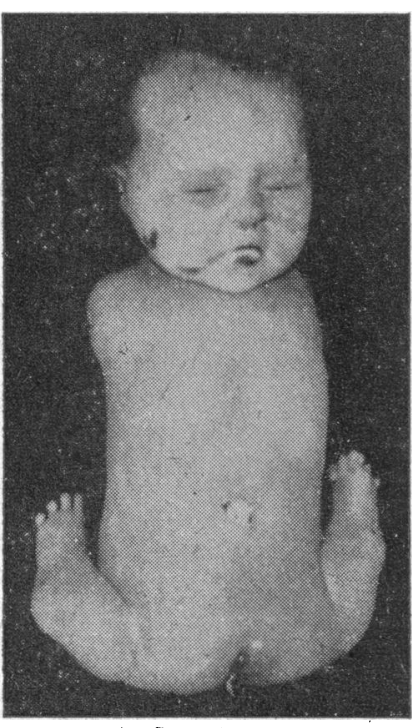

F10. 1 the pregnancy. It is of interest that the woman's general practitioner was of the opinion that without the sedative effects of thalidomide she may never have conceived at all. At full term she was delivered of a malformed female infant which died 24 hours after birth.

The post-mortem findings are summarized below.

External Examination (Fig. 1).-A female infant. Weight, $7 \mathrm{lb} .8 \mathrm{oz}$. (3.400 g.). Crown-rump length. 14 in. $(35.5 \mathrm{~cm}$.). Circumference of head, $13 \frac{3}{4}$ in. $(35 \mathrm{~cm}$.). Circumference of chest, $14 \frac{3}{4}$ in. (37.5 cm.). There was a defect in the iris of the right eye, a lens opacity in the left eye, and a complete absence of arms. The legs were rudimentary, but the feet were well developed (four digits on right, six on left).

Radiographic Examination (Fig. 2).-Skull: Normal size, shape, and development. Axial skeleton: The vertebral column was normally developed as far as the sacrum. The terminal spine was incompletely fused from S3 downwards. Thorax and shoulder-girdle: The ribs were normal in number and development and the clavicles were normal. The scapulae were hypoplastic, with no evidence of glenoid cup formation. Upper limbs: There were no ossification centres for any of the bones of the upper limbs. Pelvis : The ilia and ischia were normally developed, but there was no evidence of the pubic bones. Lower limbs: Neither

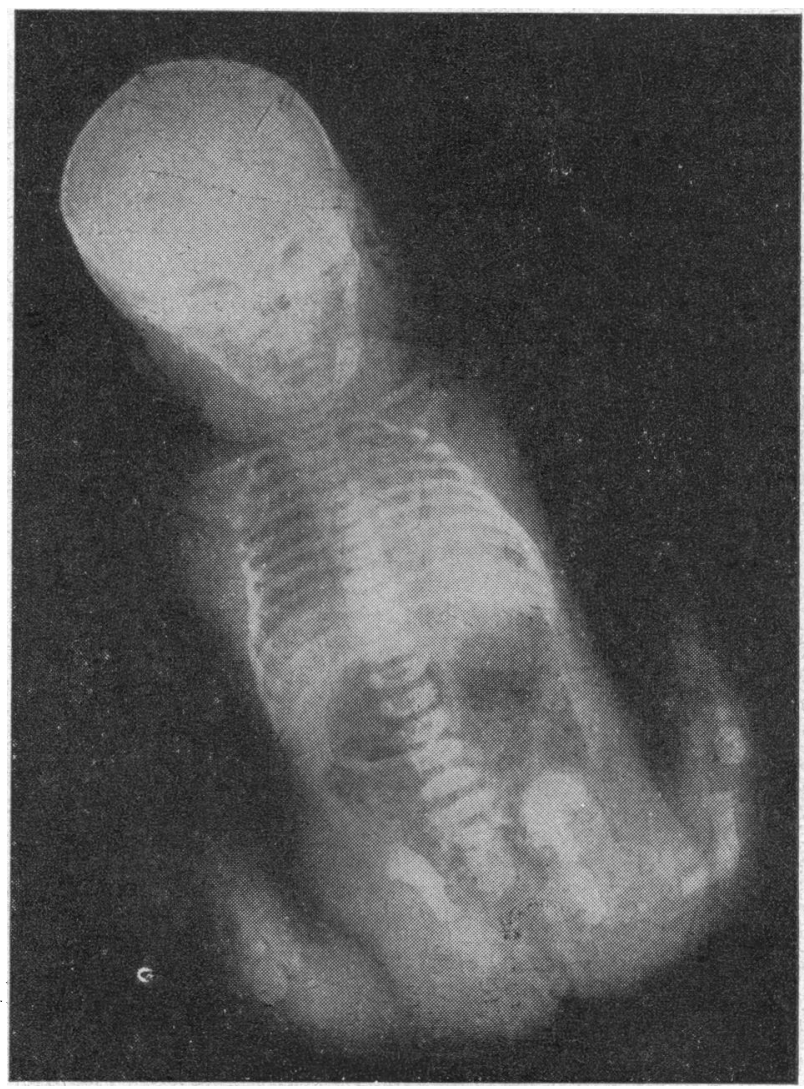

F1G. 2

femur was present. On the left there was a malformed tibia but no fibula. On the right neither tibia nor fibula was present. The tarsal bones on both sides were normalnamely, calcaneum. talus, cuboid. and lateral cuneiform. On the left foot there were five metatarsals and digits, with a supernumerary digit medial to the big toe. On the right there were four metatarsals and digits.

Internal Examination.-Respiratory system: Normal. Heart : Pericardial effusion. Dilatation of right atrium and ventricle. Persistent truncus arteriosus with a biscuspid semilunar valve arising above a high ventricular septal defect. Gastro-intestinal tract : Dilated stomach filled with meconium. Stenosis of the duodeno-jejunal junction with dilatation of the jejunum. A dilated vestigial appendix. Ileum, colon, and rectum normal. Genito-urinary tract : Normal.

Histological examination of the tissues shows no abnormality.

This case was demonstrated at the Summer Meeting of the West Mercian Branch of the Association of Clinical Pathologists, held at the Staffordshire General Infirmary. 
I wish to thank Dr. F. J. Pick, consultant pathologist, and Dr. D. A. Alderson, consultant radiologist, both of Staffordshire General Infirmary, for permission to use their reports.

BIBLIOGRAPHY

Kenny, S. (1962). Brit. J. Radiol., 35, 462.

Lenz, W. (1962). Lancet 1, 45.

McBride, W. G. (1961). Ibid., 2, 1358.

Pfeiffer, R. A., and Kosenow, W. (1962). Ibid., 1, 46.

Speirs, A. L. (1962). Ibid., 1, 303.

Woollam, D. H. M. (1962), Brit. med. J., 2, 236.

\section{THALIDOMIDE BABIES : MANAGEMENT OF LIMB DEFECTS}

BY

\section{LEON GILLIS, M.B.E., M.Ch.(Orth.), F.R.C.S. F.R.C.S.Ed., D.L.o.}

Consultant Orthopaedic Surgeon, Queen Mary's Hospital, Roehampton, S.W.15; Hon. Consultant Surgeon to the Limb Fitting Centre, Queen Mary's (Roehampton) Hospital (Ministry of Health)

From observations of the recent cases of congenital limb deformities attributed to thalidomide administered during the early weeks of pregnancy (that is, fifth and sixth weeks) it would seem that there is a fairly clearly defined clinical syndrome (see Smithells, 1962 ; Leck and Millar, 1962).

There is, commonly, a haemangioma situated centrally on the forehead. Often another is seen on the upper lip and sometimes on the cheek or the tip of the nose. These fade completely in time. Associated with this phenomenon is a depressed bridge of the nose, referred to as "saddle nose." Rarely there is microtia, and this may be associated with a partial facial palsy. Coloboma affecting one or both eyes is also sometimes seen. All the limbs are usually affected and phocomelia is the commonest type of deformity, there being various degrees from complete absence of the long bones, with one or two fingers at shoulder level, to limbs of normal length with hands having four fingers and absent thumbs or rudimentary thumbs (Figs. 1-3). This type usually has a defective or absent radius. In the upper limbs the defect always seems to be "radial "- that is, if only one finger is present it is the little one, then little and ring finger, etc. Similarly in the lower limbs there is often the long-bone defect and defects of toes, although polydactyly is not uncommon. The deformities are usually symmetrical regarding length, but often one side is slightly more developed than the other-that is, there may be three fingers on one side and two or four on the other. Upper limbs may be very short, while lower limbs are either normal or only very slightly affected. This is far more common than the reverse. More rarely, an infant is seen with bilateral amelia (absence of limb or limbs) and the other limbs normal, or with bilateral amelia and bilateral phocomelia. A single limb defect is quite rare.

There seems to be no mental retardation in the majority, although it has been reported. Defects of the cardiovascular system, urogenital system, and intestinal tract are moderately often seen.

Principles of Management.-The orthopaedic management of the limb deformities of thalidomide babies is the same as that for similar deformities due to other causes. Their treatment follows well-established principles (Gillis, 1956). It consists essentially in (1) correction of deformities and contractures by conservative means, (2) surgery to salvage badly deformed limbs, or amputation, and (3) the uses of prostheses.

\section{Conservative Correction of Deformities}

Of prime importance is the orthopaedic correction of deformities and contractures. Manipulative measures should be undertaken at a very early age in order to produce anatomical alignment and cosmetic re-position as far as possible. Serial manipulative plasters followed by plastic retentive splintage are suitable for mobile deformities. In no circumstances should amputation be done before considering what can be accomplished by ordinary orthopaedic care, by adequate splintage, and by prostheses.

The object of splintage is to correct deformities in the developing bones and joints. The object of an appliance, such as a patten, is to initiate walking and desirable movements before a semi-final decision is made regarding the desirability of supplying an artificial limb. The object of an artificial limb in these cases is (1) to conceal the deformity, (2) to extend a short limb and enable natural walking to take place, or (3) a combination of both. Such a limb is called an extension prosthesis or platform limb. It is a type of appliance which is designed to conceal deformed short limbs due to congenital abnormality, poliomyelitis, etc. The special features of the limb will vary according to the particular deformity concerned, but the general principle is as follows: a boot is constructed into which the natural foot is fitted (Fig. 4). This boot holds the foot in a position of equinus and is fixed on a platform at the required distance from the ground. The greater the degree of equinus the less obtrusive is the
Fig. 1.-Boy aged 18 months, whose mother gave a history of having taken thalidomide during early pregnancy. Note "saddle nose." and haemangioma situated on right cheek. There is a complete absence of left upper limb: on right side he has a rudimentary humerus, absent radius, and two rudimentary digits. 